\title{
THE EFFECTS OF AUGMENTED REALITY ON LEARNING PERFORMANCE AND COGNITIVE LOAD USING THE SPATIAL CONTINUITY PRINCIPLE
}

\author{
Xuewang Geng ${ }^{1}$ and Masanori Yamada ${ }^{2}$ \\ ${ }^{I}$ Graduate School of Human-Environment Studies, Kyushu University \\ ${ }^{2}$ Faculty of Arts and Science, Kyushu University \\ 744 Motooka, Nishi-ku, Fukuoka 819-0395, Japan
}

\begin{abstract}
In recent years, several studies have provided evidence for the positive impact of augmented reality in language acquisition. In particular, augmented reality as a multimedia learning environment has been found to be potentially more effective than learning using traditional methods, such as paper-based textbooks. According to the cognitive theory of multimedia learning, it can help reduce the extraneous cognitive load and improve learning by employing the spatial continuity principle. In this paper, the impact of the spatial continuity principle on the augmented reality learning system was tested by comparing the cognitive load and learning performance of two separate learning systems. The experimental results showed that the explanation integration system, which applies the principle of spatial continuity to the augmented reality learning system, was more effective in terms of knowledge retention. However, the results on the cognitive load contrasted previous research findings on the spatial continuity principle approach, as no differences were found in the extraneous cognitive load of the learners in the two systems.
\end{abstract}

\section{KEYWORDS}

Augmented Reality, Spatial Continuity Principle, Cognitive Load, Compound Verbs, Mobile Learning, Japanese Acquisition

\section{INTRODUCTION}

Advances in technology and the widespread use of smart mobile devices have transformed learning methods, making a variety of new approaches possible. Whereas traditional learning materials like textbooks are usually based on a static approach, visual technologies like augmented reality (AR) and virtual reality (VR) can interact with learners and make them experience learning contents directly (Ibáñez et al., 2016). By applying a combination of real world and virtual objects through the use of mobile devices, AR allows learners to experience them quickly and effectively without the need to wear specialized devices such as head-mounted displays.

Recently, research on the use of AR technology in education has received increasing attention and has been shown to promote motivation, increase satisfaction, make abstract or complex concepts easier to understand, and improve students' learning performance (Walczak et al., 2006; Ibrahim et al., 2018; Cabero-Almenara et al., 2019). In language acquisition, AR facilitates language learning by using more meaningful cues for the learner to see in the real world, while also integrating haptic and visual learning contents. As a multimedia learning environment, AR has been verified as being more effective for learning than text alone (Mayer, 2009). Santos et al. (2013) stated that AR learning by annotating objects is better than learning the same objects using paper-based textbooks. Therefore, AR systems have the potential to improve learning performance and knowledge retention compared to traditional learning methods, such as textbooks, in language acquisition.

The spatial continuity principle is one of the instructional design techniques for multimedia learning (Schroeder \& Cenkci, 2018). Schroeder and Cenkci (2018) indicated that the spatial continuity design helps to reduce the extraneous cognitive load and improve learning. AR can easily integrate learning contents and additional information by attaching virtual content to learning objects. In contrast, other ineffective instructional designs may lead to distraction effects and thus increase the extraneous cognitive load (Sweller, 
2010). This paper compares the learning performance and cognitive load of two AR systems to verify the effect of the spatial continuity principle.

This paper is organized as follows: a literature review on AR systems in language learning and the spatial continuity principle is conducted in Section 2. Section 3 presents the two AR learning systems and describes the research questions of this study. Section 4 justifies the methodology of this research. Section 5 describes the results and discusses these findings. Section 6 concludes the paper and proposes future lines of research.

\section{LITERATURE REVIEW}

\subsection{AR Systems in Language Acquisition}

Azuma (1997) defined three features of an AR system: the combination of real and virtual, real-time interaction, and being registered in 3D. In education, these features provide three main advantages: annotations of the real world, visualizations of context, and visualizations of vision-haptic (Santos et al., 2013). In turn, these advantages have the potential to facilitate employing AR in language acquisition. Boonbrahm et al. (2015) developed an AR application for learning the spellings and meanings of animal words. In the application, the learners can arrange letters to form a word that matches the name of the animal, and the 3D animal will be displayed on the application. Santos et al. (2016) developed and designed the two handheld AR systems for foreign vocabulary learning by using the surrounding spatial information. Moreover, Hsu (2017) developed the AR educational game to help third grade students learn English vocabulary. As such, many studies have concluded that AR systems have the potential to improve learning performance, as well as knowledge retention in language acquisition (e.g., Ibrahim et al., 2018; Hsu, 2017; Santos et al., 2016; Boonbrahm et al., 2015).

AR systems can be categorized into location-based and image-based systems. Location-based systems refer to the use of mobile devices' location services in relation to the recognition function. Image-based AR systems focus on image-recognition technology, and use real images as marks to identify objects that virtual information is attached to. Whether using the location-based or image-based AR system, Santos et al. (2013) stated that AR facilitates interaction with information, which assists learners in perceiving and remembering information.

In the specific case of the Japanese language, compound verbs are difficult learning items even for advanced learners (Matsuda, 2000). Japanese compound verbs are similar to phrasal verbs in English, and they are formed from two single verbs (see Figure 1; e.g., the compound verb tori-hazusu is formed from the single verbs toru and hazusu). The AR learning system was designed and developed to facilitate learning Japanese compound verbs based on the difficulties of compound verb acquisition (Geng \& Yamada, 2019). It is an image-based AR system that displays the meaning of the verb with virtual information on the real verb characters.

\subsection{The Spatial Continuity Principle}

According to the cognitive theory of multimedia learning, since human working memory is limited, learners should have the cognitive ability to select information, organize relevant information, and integrate it within prior knowledge structures (Mayer, 2014). Consistent with the cognitive load theory (Sweller, 2010), Mayer (2014) indicated that there are three types of cognitive loads that arise when learners engage in cognitive processing during multimedia learning, namely, extraneous cognitive load, intrinsic cognitive load, and germane cognitive load. The intrinsic cognitive load is related to the complexity of information, the extraneous cognitive load is related to the design of materials, and the germane cognitive load is related to the acquisition of knowledge (Sweller, 2010).

Instructional design and learning materials affect learners' cognitive processes, thus interacting with the learning environment and the cognitive load experience (Paas \& Sweller, 2014). The spatial continuity principle is one of the instructional design techniques developed using the cognitive theory of multimedia learning as a theoretical foundation (Schroeder \& Cenkci, 2018). The principle of spatial continuity proposes that people learn more from multimedia messages when the corresponding words and pictures are close to each other on the page or screen rather than far away from each other, as this avoids the split-attention effect (Mayer $\&$ Fiorella, 2014). To solve the spatial separation, the physical distance between relevant information should be reduced by displaying the relevant information near the learning contents. In language acquisition, since the 
AR features real-world annotations, words and visual information can be easily integrated to display them in the spatial vicinity. However, there are some contradictory results in terms of the spatial continuity principle. Schroeder and Cenkci (2018) indicated that spatial continuity design helps to reduce extraneous cognitive load and improve retention of learning. In spite of this, there are some studies that did not find the benefits of the spatial continuity principle (e.g., Florax and Ploetzner, 2010). Therefore, it is crucial to probe the cognitive load and learning performance of the AR learning system design based on the spatial continuity principle.

\section{THE AR COMPOUND VERB LEARNING SYSTEM}

In our previous research (Geng \& Yamada, 2019; 2020), an AR learning system was designed and developed to facilitate the acquisition of Japanese compound verbs. Chen (2007) indicated that there are some difficulties in Japanese compound verb acquisition such as opacity in meaning: learners have difficulties understanding the meaning of compound verbs, and distinguishing between the meaning of compound verbs and the single verbs that form them. Therefore, the system is grounded on marker-based AR and shows learners the meaning of the verbs through $3 \mathrm{D}$ animations. In this system, learners can scan the verb cards and the animations of the verbs' meanings will be displayed on the card via the smartphone screen (see Figure 1). There are 11 cards printed with the characters of the single verb, which can be combined to form 8 compound verbs. In other words, learners learn single verbs by scanning the verb cards first, and then learn compound verbs consisting of two single verbs by correctly combining the two verb cards. This allows them to distinguish between a single verb and a compound verb by scanning and combining the cards to compare their meanings.

Regarding the meaning of verbs, animations as well as explanations and example sentences of the verbs are used to supplement verb learning in the system. Since the animations of verbs in this AR system (explanation separation system, hereafter referred to as ES) are spatially separated from the verb explanation materials, there are spatial split-attention effects in the system. We also developed a new system (explanation integration system, hereafter referred to as EI) based on the spatial continuity principle using the ES system to display verb explanations and animations on the same screen.

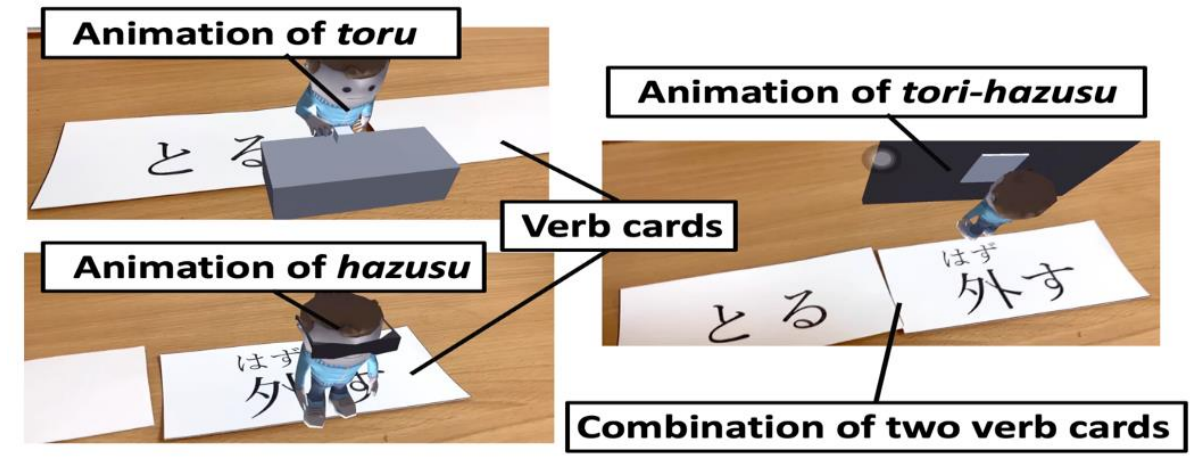

Figure 1. The verb card combinations of the AR learning system

\subsection{ES System}

In the ES system, verb explanations are provided to learners as paper-based learning materials to help them better understand the animation of verbs, and thereby learn compound verbs. As there is a spatial separation between the verb explanations and the animations, it is necessary to keep the verb cards on the screen while seeing the animations (see the image on the left in Figure 2). However, it is difficult for learners to refer to the learning materials while seeing the animations. In practice, learners tend to read the learning material before or after viewing the animations. 


\subsection{EI System}

In contrast to the ES system, the EI system displays explanations of meanings on the screen of the smartphone. When the learners correctly scan the cards or combine the verb cards to form compound verbs, the explanations and animation of the compound verb meaning will be displayed on the screen (see the image on the right of Figure 2). The explanations of the verbs in the EI system is identical to the content of the paper-based learning materials in the ES system. The explanations and animations are both triggered at the same time. Therefore, learners must keep the verb cards on the screen while seeing the animations and explanations.

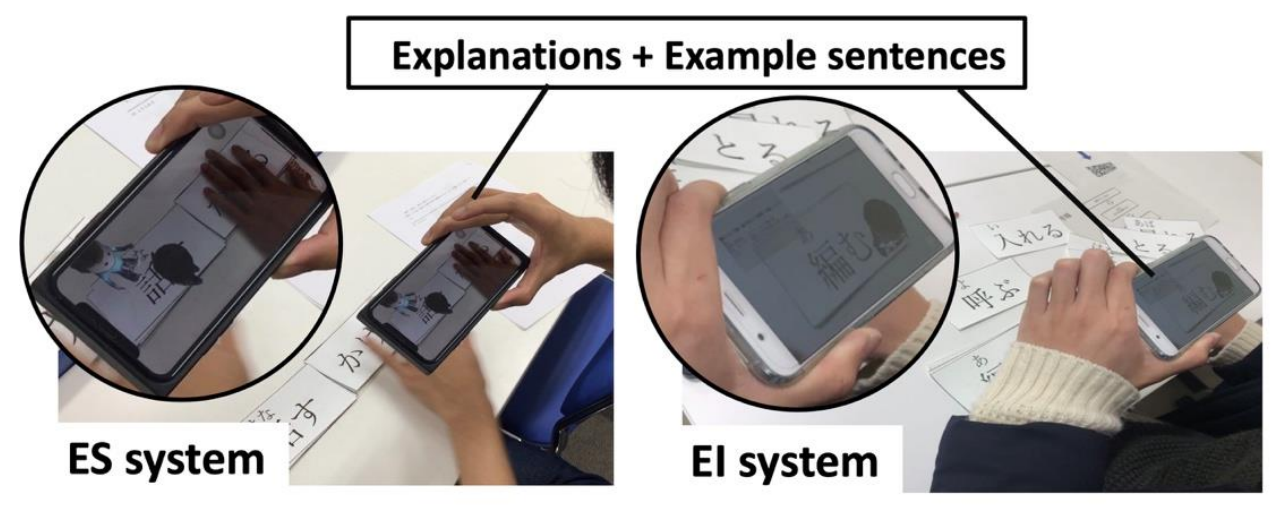

Figure 2. The usage of the ES system and the EI system

\subsection{Research Questions}

In this study, we investigated the effect of the spatial continuity principle by comparing the learning performance and cognitive load of these two AR language learning systems. For this purpose, we set the following two research questions.

RQ1: How does the learning performance of the EI system compare to the ES system?

RQ2: How does the perceived cognitive load of the EI system compare to the ES system?

\section{METHODOLOGY}

\subsection{Participants}

Two experiments were conducted in this study on the ES system and the EI system, respectively. The first experiment recruited ten participants and the second recruited thirteen participants, such that there were 23 participants in total. The participants were non-native Japanese speakers from China, Korea, Vietnam, Thailand, Cambodia, and other countries. However, they had an upper intermediate level of Japanese language proficiency, which meant that they could understand the explanations of the verbs provided in the experiments. Five male and five female participants took part in Experiment 1 and seven males and six females were included in Experiment 2. The age of all participants ranged from 18 to 30 years old.

\subsection{The Experimental Procedure}

Both Experiments 1 and 2 were 90-minutes long, and Experiment 2 was implemented a few months after Experiment 1. Both experiments were conducted using the same learning content and aimed to facilitate Japanese compound verb learning. 
The procedure was basically the same for both experiments as shown in Figure 3. First, all participants were tested prior to the learning activity in order to assess their knowledge of compound and single verbs. All participants performed the learning activity for 40 minutes. However, participants in Experiment 1 used the learning material and the ES system to learn by scanning and combining cards, whereas participants in Experiment 2 did not need paper-based learning material during the learning activity. At the end of the learning activity, all participants took another test and the cognitive load questionnaire. Four weeks later, participants took a delayed test in Experiment 1. Due to COVID-19, participants in Experiment 2 took the delayed test only one week after the post-test.

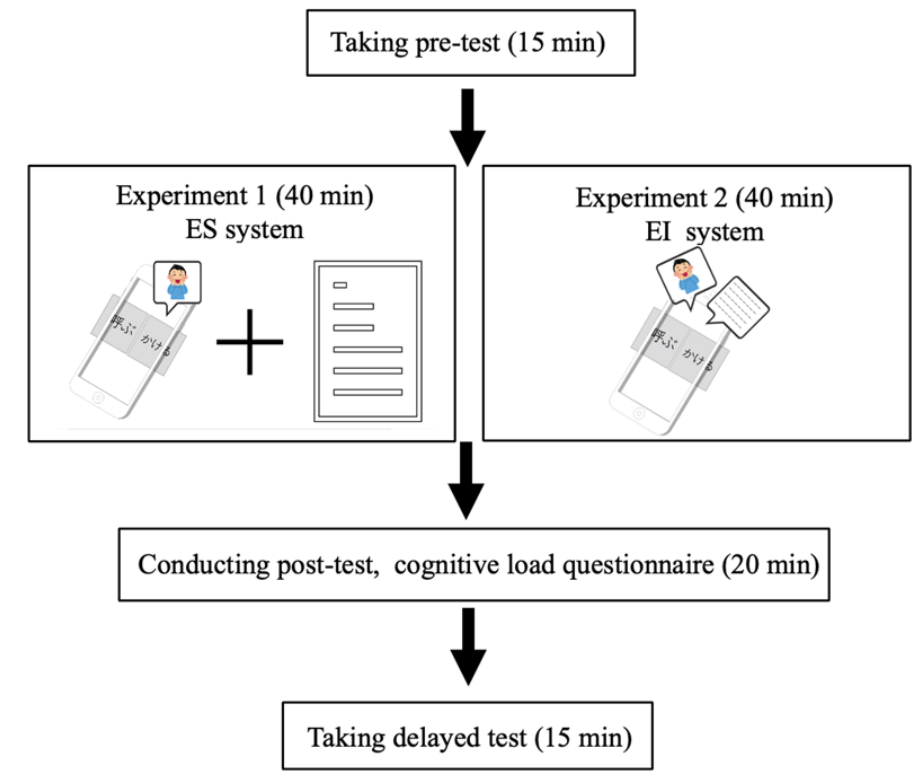

Figure 3. The procedure of the two experiments

\subsection{Measuring Tools}

The measuring tools in the study were composed of the pre-test, post-test, delayed test, and the cognitive load questionnaire. The pre, post, and delayed tests contained the same questions and options, but were presented in a different order. The tests consisted of two parts including fifteen true-or-false questions to judge the correct or incorrect options of the compound verbs and twenty-six multiple-choice questions to test the meaning of verbs. Each question was worth 1 point, and the total score for each test was 41 points.

The cognitive load questionnaire employed in this study was created by Leppink et al. (2013). The questionnaire measures the perceived cognitive load during the learning activity: intrinsic cognitive load, extraneous cognitive load, and germane cognitive load. It was performed using an 11-level Likert scale for ten questions ( 0 for completely disagree and 10 for completely agree). The intrinsic cognitive load consisted of three items on whether the compound verbs covered in the activity were complex. The extraneous cognitive load consisted of three questions on whether the explanations and AR system were clear and effective learning materials. Furthermore, there were four questions on the germane cognitive load (e.g., "The activity really enhanced my knowledge and understanding"). 


\section{EXPERIMENTAL RESULTS AND DISCUSSION}

\subsection{How does the Learning Performance of the EI System Compare to the ES System?}

The summary statistics for mean values, median values, and standard deviations of the two experiments' total scores on the pre-test, post-test, and delayed test are shown in Table 1. From Table 1, it appears that the pre-test scores of Experiment 1 were slightly higher than Experiment 2. Since the sample size was small and not normally distributed, non-parametric analyses were conducted in this study. Therefore, the Mann-Whitney $\mathrm{U}$ test was performed on the pre-tests of the two experiments to examine whether there was a difference in the participants' prior knowledge of compound verbs. There was no statistically significant difference of the pre-tests of the two experiments $(|\mathrm{Z}|=1.367$, n.s.). In addition, post and delayed test scores were higher in both experiments than in the pre-test.

The results of the two experiments showed that learners who used the AR learning systems of ES and EI improved their learning performance. These results further support the idea that the AR system is able to facilitate learning Japanese compound verbs.

Table 1. Mean, median, and SD values of the total scores in the three tests of the two experiment

\begin{tabular}{lllll}
\hline Tests (total score: 41$)$ & Experiment & Mean & Median & SD \\
\hline \multirow{2}{*}{ Pre-test } & Experiment 1 & 29.10 & 29.00 & 5.80 \\
& Experiment 2 & 25.85 & 26.00 & 4.63 \\
\hline \multirow{2}{*}{ Post-test } & Experiment 1 & 34.20 & 35.50 & 4.08 \\
& Experiment 2 & 33.08 & 34.00 & 1.98 \\
\hline \multirow{2}{*}{ Delayed test } & Experiment 1 & 31.90 & 32.00 & 5.22 \\
& Experiment 2 & 33.69 & 34.00 & 4.25 \\
\hline
\end{tabular}

We also conducted Mann-Whitney $U$ tests on the change in test scores between the two experiments in order to verify the differences in learning performance using the ES and EI systems. The results of the Mann-Whitney U tests are shown in Table 2. Regarding the changes of scores between the post-test and the pre-test (post-test minus pre-test), no statistically significant differences were found between the two experiments. Conversely, there were significant differences in score changes between delay-pretests, and post-delay tests (changes in delayed test and pre-test scores : $|\mathrm{Z}|=2.434, \mathrm{p}<.05$; changes in post-test and delayed test scores $:|Z|=2.190, \mathrm{p}<.05)$.

Table 2. The Mann-Whitney U test results on changes in test scores

\begin{tabular}{lllllll}
\hline \multirow{2}{*}{ Changes in test scores } & \multicolumn{2}{l}{ Experiment $1(\mathrm{n}=10)$} & \multicolumn{2}{l}{ Experiment 2 $(\mathrm{n}=13)$} & \multirow{2}{*}{$\mathrm{Z} \mid$} & \multirow{2}{*}{$\mathrm{p}$-value } \\
\cline { 2 - 6 } & Mean & $\mathrm{SD}$ & Mean & SD & & \\
\hline Post - Pre & 5.10 & 3.54 & 7.23 & 3.96 & 1.249 & 0.223 \\
\hline Delay -Pre & 2.80 & 3.43 & 7.85 & 4.95 & $2.434^{*}$ & 0.016 \\
\hline Post- Delay & 2.30 & 2.79 & -0.62 & 3.25 & $2.190^{*}$ & 0.031 \\
\hline$\dagger \mathrm{p}<1,{ }^{*} \mathrm{p}<.05,{ }^{* * \mathrm{p}<.01}$ & & & & & &
\end{tabular}

As such, the current study found that learners using the EI system had greater changes between the delay-pretest, and smaller changes between post-delay test than learners using the ES system. Regarding the results of the change in test scores, it can be concluded that the EI system is more effective in terms of learners retaining knowledge. This finding supports evidence from the spatial continuity principle (Mayer, 2014). The EI system physically integrates the text into the visual display, reducing the split attention on the two information sources of verb explanation and animation compared to the ES system. This finding is contrary to previous studies which noted that interactive learning materials using the spatial continuity principle do not benefit as much as static and dynamic materials from spatial continuity design (Schroeder \& Cenkci, 2018). However, the most important limitation lay in the different time intervals between the post-test and delayed test of the two experiments, due to the COVID-19 outbreak. Further research should control the time interval for consistency. 


\subsection{How does the Perceived Cognitive Load of the EI System Compare to the ES System?}

The cognitive load questionnaire employed in this study measured the intrinsic cognitive load, extraneous cognitive load, and germane cognitive load, which the learners perceived using the ES or EI system. We analyzed the collected questionnaires and calculated three types of cognitive loads according to the answers of the related questions. The Mann-Whitney U-tests were performed to investigate the influence of ES and EI systems on the perceived cognitive load. The results are shown in Table 3. No statistically significant difference was found between the two experiments in terms of extraneous and germane cognitive load. However, it was found that the intrinsic cognitive load of Experiment 2 was higher than Experiment 1 at the significance value of 0.1 .

Surprisingly, no differences were found between the extraneous cognitive load of the ES and EI system. Therefore, this finding was incompatible with the spatial continuity principle (Mayer, 2014). This study was unable to demonstrate that learners perceive less extraneous cognitive load using an AR system designed on the principle of spatial continuity. In contrast, there was a significant trend on intrinsic cognitive load of ES and EI system learners. The intrinsic cognitive load is determined by the complexity of the learning content and the learner's prior knowledge (Sweller, 2010). Based on a review of existing literature, Mayer and Fiorella (2014) concluded that the spatial continuity principle is most effective for learners with low prior knowledge. Although there was no difference between pre-test scores, there is indirect evidence supporting the fact that learners using the EI system retain knowledge better, as the intrinsic cognitive load of EI system learners was found to be higher. However, these results need to be explored further, because our experiments involved a low sample size. Furthermore, there is still a limitation in psychometric measures of cognitive load, since it is difficult for learners to clearly distinguish between different types of cognitive load (Schnotz \& Kürschner, 2007).

Table 3. The Mann-Whitney U test results on cognitive load

\begin{tabular}{lllllll}
\hline \multirow{2}{*}{ Cognitive Load } & \multicolumn{2}{l}{ Experiment $1(\mathrm{n}=10)$} & \multicolumn{2}{l}{ Experiment 2 $(\mathrm{n}=13)$} & \multirow{2}{*}{$|\mathrm{Z}|$} & \multirow{2}{*}{$\mathrm{p}$-value } \\
\cline { 2 - 6 } & Mean & $\mathrm{SD}$ & $\mathrm{Mean}$ & $\mathrm{SD}$ & & \\
\hline Intrinsic cognitive load & 4.67 & 2.23 & 6.31 & 1.54 & $1.926^{\dagger}$ & 0.058 \\
\hline Extraneous cognitive load & 1.43 & 1.48 & 2.13 & 1.89 & 1.068 & 0.300 \\
\hline Germane cognitive load & 7.66 & 1.63 & 7.27 & 1.29 & 0.281 & 0.803 \\
\hline $\mathrm{p}<1,{ }^{*} \mathrm{p}<.05, * * \mathrm{p}<.01$ & & & & & &
\end{tabular}

\section{CONCLUSION AND FUTURE WORK}

In this study, researchers explored the effects of the spatial continuity principle on the AR learning system by comparing the cognitive load and learning performance of two learning systems. We developed the EI system design based on the spatial continuity principle using the ES system. Two experiments were conducted to separately verify each system. The results of the experiments confirmed that learners using the EI system are more effective at retaining knowledge than learners of the ES system. This study also showed that there was a significant trend on intrinsic cognitive load, and no differences were found in the extraneous cognitive load of ES and EI system learners. However, the results need to be explored further by testing over a longer period of time and with a larger sample size. Furthermore, instead of relying on questionnaires to measure the cognitive load, other approaches should be used in future research. For example, behavioral assessments using learning analytics is a useful approach to investigating the relationships between learning processes and learning performances in terms of learner behaviors (e.g., Okubo et al, 2016; Kaneko et al, 2018).

\section{ACKNOWLEDGEMENT}

This research was supported in JSPS kaken-hi, Grant.No. JP18K18657, JP19H01716. 


\section{REFERENCES}

Azuma, R. T., 1997. A survey of augmented reality. Teleoperators \& Virtual Environments, Vol .6, No. 4, pp. 355-385.

Boonbrahm, S. et al, 2015. Using augmented reality technology in assisting English learning for primary school students. In International Conference on Learning and Collaboration Technologies, pp. 24-32.

Cabero-Almenara, J. et al, 2019. Adoption of augmented reality technology by university students. Heliyon, Vol. 5, No. 5, pp. 1-9.

Chen, X. 2007. Nihongo fukugoudousi no syutokujyoukyou to shidou hei no mondaiteiki (The acquisition of Japanese compound verbs and implications for teaching: An examination of "au" and "komu" at Xi' an Foreign Language University). Forum of International Development Studies, Vol. 35, No. 1, pp. 93-102.

Florax, M., and Ploetzner, R., 2010. What contributes to the split-attention effect? The role of text segmentation, picture labelling, and spatial proximity. Learning and instruction, Vol. 20, No. 3, pp. 216-224.

Geng, X., \& Yamada, M., 2020. An augmented reality learning system for Japanese compound verbs: study of learning performance and cognitive load. Smart Learning Environments, Vol.7, No.1, pp. 1-19.

Geng, X., and Yamada, M., 2019. Development and design of a compound verb AR learning system employing image schemas. In 15th International Conference on Mobile Learning 2019, ML 2019, pp. 73-80.

Hsu, T. C., 2017. Learning English with augmented reality: Do learning styles matter? Computers \& Education, Vol. 106, pp. 137-149.

Ibáñez, M. B. et al, 2016, The acceptance of learning augmented reality environments: A case study. In 2016 IEEE 16th International Conference on Advanced Learning Technologies (ICALT), Austin, USA, pp. 307-311.

Ibrahim, A. et al, 2018. Arbis pictus: A study of vocabulary learning with augmented reality. IEEE Transactions on Visualization and Computer Graphics, Vol. 24, No. 11, pp. 2867-2874.

Kaneko, K. et al, (2018). Does physical activity enhance learning performance?: Learning effectiveness of game-based experiential learning for university library instruction. The Journal of Academic Librarianship, 44(5), pp. 569-581

Leppink, J. et al, 2013. Development of an instrument for measuring different types of cognitive load. Behavior research methods, Vol. 45, No. 4, pp. 1058-1072.

Matsuda, F. 2000. Fukuguodoushi no imi rikai houryaku no jittai to syutoku konnanten (Acquisition of compound verbs in Japanese by advanced learners of Japanese: Learners' strategies of comprehending the meanings of compound verbs). Japanese Language Education, Vol. 20, No. 1, pp. 52-65.

Mayer, R. E. 2014. The Cambridge handbook of multimedia learning (2nd ed.). Cambridge University Press, New York, USA

Mayer, R. E., 2009. Multimedia learning (2nd ed.). Cambridge University Press, New York, USA.

Mayer, R., and Fiorella, L., 2014. Principles for Reducing Extraneous Processing in Multimedia Learning: Coherence, Signaling, Redundancy, Spatial Contiguity, and Temporal Contiguity Principles. In R. Mayer (Ed.), The Cambridge Handbook of Multimedia Learning (Cambridge Handbooks in Psychology, pp. 279-315). Cambridge University Press, Cambridge, UK.

Okubo, F. et al, 2016, Learning Activity Features of High Performance Students, Proc. of the 1st International Workshop on Learning Analytics Across Physical and Digital Spaces (Cross-LAK 2016), Edinburgh, UK, pp. 28-33

Paas, F., and Sweller, J., 2014. Implications of cognitive load theory for multimedia learning. The Cambridge Handbook of Multimedia Learning, vol. 27, pp. 27-42.

Santos, M. E. C. et al, 2013. Augmented reality learning experiences: Survey of prototype design and evaluation. IEEE Transactions on Learning Technologies, Vol. 7, No. 1, pp. 38-56.

Santos, M. E. C. et al, 2016. Augmented reality as multimedia: The case for situated vocabulary learning. Research and Practice in Technology Enhanced Learning, Vol. 11, No. 4, pp.1-23.

Schnotz, W., and Kürschner, C., 2007. A reconsideration of cognitive load theory. Educational psychology review, Vol. 19, No. 4, pp. 469-508.

Schroeder, N. L., and Cenkci, A. T. 2018., Spatial contiguity and spatial split-attention effects in multimedia learning environments: A meta-analysis. Educ Psychol Rev, Vol. 30, pp. 679-701

Sweller, J. 2010., Element interactivity and intrinsic, extraneous, and germane cognitive load. Educational Psychology Review, Vol. 22, No. 2, pp. 123-138.

Walczak, K. et al, 2006. Dynamic interactive VR network services for education. In Proceedings of the ACM symposium on Virtual reality software and technology, pp. 277-286. 